\title{
APORTES TEÓRICOS PARA PENSAR EL TRABAJO DOCENTE EN LA UNIVERSIDAD \\ THEORETICAL CONTRIBUTIONS TO THINK UNIVERSITY TEACHING WORK
}

\author{
Volumen 15, Número 1 \\ Enero - Abril \\ pp. $1-20$
}

Este número se publicó el $1^{\circ}$ de enero de 2015

DOI: dx.doi.org/10.15517/aie.v15i1.16964

Verónica Soledad Walker

Revista indizada en REDALYC, SCIELO

Revista distribuida en las bases de datos:

CATÁLOGO DE LATINDEX, IRESIE, CLASE, DIALNET, DOAJ, E-REVIST@S, SHERPA/ROMEO, QUALIS, MIAR

Revista registrada en los directorios:

ULRICH'S, REDIE, RINACE, OEI, MAESTROTECA, PREAL, CLACSO

Los contenidos de este artículo están bajo una licencia Creative Commons

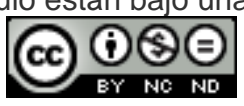




\title{
APORTES TEÓRICOS PARA PENSAR EL TRABAJO DOCENTE EN LA UNIVERSIDAD \\ THEORETICAL CONTRIBUTIONS TO THINK UNIVERSITY TEACHING WORK
}

\section{Verónica Soledad Walker ${ }^{1}$}

Resumen: Este ensayo pretende aportar elementos teóricos que contribuyan a la comprensión del trabajo docente universitario. A partir del análisis de la bibliografía especializada y de información recogida por medio de entrevistas realizadas a docentes universitarios argentinos y españoles, se abordan cuestiones referidas a las condiciones materiales y simbólicas, la identidad y la práctica docente. Primero, se analiza la docencia universitaria desde la categoría de trabajo, reconociendo las condiciones mencionadas en las que este se desarrolla. Segundo, se aborda el trabajo docente como el entrecruzamiento entre una profesión de origen y la actividad de docencia. Tercero, se plantea la multidimensionalidad que hace de este trabajo una práctica compleja. El análisis realizado permite afirmar que en ambos contextos geográficos el trabajo docente se ha intensificado, diversificado y es minusvalorado en relación con la investigación; mientras el reconocimiento de los pares opera como fuente de satisfacción. La identidad profesional permite reconocer la diversidad de perfiles docentes y la desigualdad en términos de saberes pedagógicos y prestigio. Las dimensiones estudiadas abonan en la configuración del trabajo docente universitario como una práctica social compleja, específica y heterogénea que en sus prescripciones, regulaciones y mecanismos de evaluación es tratada de manera homogénea.

Palabras clave: UNIVERSIDAD, TRABAJO DOCENTE, CONDICIONES DE TRABAJO, ORGANIZACIÓN DEL TRABAJO, DISCIPLINAS ACADÉMICAS, IDENTIDAD DOCENTE

\begin{abstract}
This essay proposes to provide theoretical elements that contribute to the understanding of university teaching. Based on the analysis of the literature and information gathered through interviews with Argentine and Spanish academics, issues relating to the conditions, identity and teaching practice are discussed. First, university education is analyzed from the work category, recognizing the material and symbolic conditions in which it develops and the dimensions that cross it. Second, the work of teachers as a profession interbreeding between the source profession and the activity of teaching is addressed. Third, the multidimensionality that makes college teaching work a complex practice is approached. The analysis allows us to assert that in both contexts the teaching work has been intensified, diversified and is undervalued in relation to the investigation; while peer recognition operates as a source of satisfaction. The disciplinary dimension allows us to recognize the diversity of teacher profiles and inequality in terms of pedagogical knowledge and prestige. The dimensions prescribed and actual configuration recognize university teaching job as a complex, heterogeneous and specific social practice while in requirements, regulations and evaluation mechanisms is treated uniformly.
\end{abstract}

Keywords: UNIVERSITY, TEACHING WORK, WORKING CONDITIONS, WORK ORGANIZATION, ACADEMIC DISCIPLINES, TEACHING IDENTITY

\footnotetext{
${ }^{1}$ Docente-investigadora de la Universidad Nacional del Centro de la Provincia de Buenos Aires, Argentina. Doctora en 'Didáctica y Organización Educativa', Universidad de Málaga, España. Especialista en Ciencias Sociales y Humanidades, Universidad Nacional de Quilmes, Argentina; Profesora y Licenciada en Ciencias de la Educación (UNCPBA).
}

Dirección electrónica: veroswalker@gmail.com

Ensayo recibido: 8 de abril, 2014

Enviado a corrección: 29 de julio, 2014

Aprobado: 20 de octubre, 2014 


\section{Introducción}

Este ensayo pretende aportar elementos teóricos que contribuyan a la comprensión del trabajo docente universitario como una práctica compleja, heterogénea y específica. A partir del análisis de la bibliografía especializada en el tema y de la información recogida a través de entrevistas efectuadas a docentes universitarios, ${ }^{2}$ se abordan cuestiones que resultan centrales en el estudio de la docencia en la universidad.

Una mirada a la bibliografía especializada en temas de docencia universitaria, permite advertir que bajo dicha expresión se aglutinan diversos sentidos y niveles de análisis. En líneas generales, la categoría 'docencia universitaria' es empleada para hacer referencia a prácticas, tanto a nivel de las instituciones como de los sujetos. Por un lado, la docencia se suele presentar como una de las funciones de las instituciones universitarias, junto con la investigación y la extensión. Por otro, se la entiende como las actividades que desarrollan determinados sujetos en el ejercicio de su función docente. En algunos casos se la circunscribe a tareas de enseñanza y en otros, se contemplan varias actividades.

En esta comunicación se aborda la docencia universitaria desde el nivel de los sujetos, entendiéndola como una actividad que incluye la de enseñanza, pero que no se reduce a ella. De este modo, lo que interesa analizar es la práctica que desarrollan determinados sujetos en el ejercicio de su función docente en la universidad. Tales sujetos suelen ser denominados como docentes, profesores, catedráticos, etc. Cuando se consideran también sus actividades de investigación, se los suele designar con el término de académicos 0 miembros de la profesión académica. Si bien aquí no se adoptará tal denominación -por lo que no se profundizará en ella, ${ }^{3}$ - sí se señalará cierta tendencia presente en las últimas décadas en el campo académico, en relación con dichos sujetos. Se trata de cambios que han transformado el tradicional ejercicio del trabajo académico en la universidad, como práctica que complementaba y legitimaba el desempeño de una carrera profesional en el propio mercado ocupacional (la de abogado, médico, contador, etc.). Precisamente, con la expansión, diversificación y diferenciación de los sistemas de educación superior durante las últimas décadas del siglo XX, se conforma un amplio mercado ocupacional -entendido como un conjunto de posiciones y condiciones de trabajo que se ofrecen y de criterios,

\footnotetext{
${ }^{2}$ Se entrevistó a 69 docentes de distintas áreas disciplinares, categoría y dedicación docente, pertenecientes a dos universidades argentinas y una española, durante el periodo 2012/2013. Se trató de un trabajo de investigación financiado por la Unión Europea en el marco del Programa Erasmus Mundus 'Move on Education', Action 2, EACEA/29/09 Lote 13b.

${ }^{3}$ Desde distintas perspectivas, autores como P. Altbach, J. J. Brunner, B. Clark, A. Chiroleu y A. García de Fanelli abordan el tema de la profesión académica.
} 
procedimientos y mecanismos que regulan su ocupación y renovación- en el cual las tareas académicas constituyen para muchos graduados, un fin en sí mismas. En este contexto, la docencia universitaria, sostiene Chiroleu (2002), se constituye como una categoría residual, formada por graduados de todas las disciplinas y, muy especialmente, de aquellas cuyo campo ocupacional extrauniversitario resulta más recortado y complejo. Es en la docencia universitaria como actividad laboral, como el trabajo que realizan cotidianamente los profesores en la universidad, sobre lo que se focaliza en los siguientes apartados, con independencia de que se desempeñen o no otras actividades laborales. ${ }^{4}$

No se pretende aquí alcanzar una definición de lo que significa la docencia en la universidad -sus peculiaridades lo tornarían difícil, cuanto no imposible; además, entrañaría el riesgo de caer en simplificaciones- pero sí plantear determinadas cuestiones que no pueden perderse de vista al estudiar dicha práctica. La primera cuestión por destacar tiene que ver con considerar la docencia universitaria como trabajo. La segunda, entenderla como el entrecruzamiento entre una profesión de origen y la actividad de docencia. La tercera, concebirla como una práctica compleja. Se trata de cuestiones que remiten a los temas de las condiciones, la identidad y la práctica docente, respectivamente.

\section{La docencia como trabajo}

Al igual que hace más de diez años Martínez Bonafé (1999) lo planteara para los estudios de la docencia en el nivel primario, en la actualidad puede decirse que la academia pedagógica, en general no piensa las actividades que desarrolla el profesor universitario desde la categoría de trabajo. Por el contrario, en la bibliografía sobre el tema se advierte que la mayoría de los estudios (tomando como punto de partida el carácter profesional de la docencia y reconociendo su complejidad -término que muchas veces sirve de comodín para lo que es difícil enunciar-) se abocan a describir experiencias catalogadas como 'exitosas', a enumerar un conjunto de 'buenas prácticas' o -más recientemente- a identificar y definir las 'competencias' necesarias para llevar adelante una 'docencia de calidad'. Ante este panorama, en el presente texto se pretende tomar distancia de aquellas tradiciones ideológicas que han mediatizado la labor docente, influyendo en ella y tornando invisibles

\footnotetext{
${ }^{4}$ Como podrá verse a lo largo del trabajo, la docencia universitaria no constituye una categoría homogénea. De ahí que en el campo universitario se puedan encontrar sujetos dedicados exclusivamente a la actividad académica, otros enfocados únicamente a tareas docentes; también están aquellos que reparten su tiempo entre la labor académica y el ejercicio de su profesión liberal. Tales situaciones junto a las diferentes dedicaciones (exclusiva, semiexclusiva y simple o completa, parcial y simple -en el sistema universitario argentino o español, respectivamente-) dan lugar a una gama heterogénea de realidades.
} 
sus condiciones materiales y simbólicas. Precisamente, al considerar la docencia universitaria como trabajo, se pretende romper con la ideología del profesionalismo como ideal que "hace que las funciones reales del trabajo, su práctica y las relaciones sociales queden oscurecidas" (Densmore, 1990, p. 124). No se trata de discutir si la docencia es una profesión o no, sino más bien de realizar ciertas advertencias respecto de la 'ideología del profesionalismo' que subyace en numerosos estudios sobre la docencia universitaria.

El carácter profesional de la docencia ha sido muy debatido, fundamentalmente en los niveles educativos primario y secundario. El enfoque clásico desde el cual se abordaron las discusiones, es aquel que entiende las profesiones como un conjunto de determinadas características. De allí que, de acuerdo con lo que se defina como un profesional -en función de un conjunto de rasgos- se determina si a una ocupación le corresponde o no tal denominación. Cuando se ha comparado a los enseñantes con las características que hacen a una profesión, la conclusión general ha sido que la docencia es una semiprofesión (por carecer de autonomía respecto del Estado, un conocimiento especializado propio y una organización monopolista propia que regule su acceso y código profesional). Sostiene Contreras (2011) que la debilidad de esta teoría reside en el simplismo de su punto de partida, ya que se identifica lo que es una profesión, con elementos seleccionados a priori, introduciendo de esta manera un sesgo ideológico, por cuanto se presenta como descriptivo lo que no deja de ser una selección interesada de elementos. Así, la teorización de los rasgos constituye una formalización de determinados supuestos ideológicos. En el caso de la educación superior, el carácter profesional de los docentes no ha sido puesto en discusión, lo cual puede entenderse en el marco del poder legitimador de las instituciones de las que forman parte. Como sostiene Bourdieu (2008), la universidad es una institución que se arroga para sí el monopolio de la verdad sobre sí y el mundo social. Sin embargo, aunque no se ponga en cuestión su estatuto de profesionales, no todas las actividades que realizan los docentes universitarios son reconocidas de la misma manera, pues las actividades de investigación gozan de mayor prestigio y reconocimiento que las tareas de docencia y extensión.

Como afirma Contreras (2011), la cuestión del profesionalismo constituye un debate vigente en el campo educativo, ya sea como expresión de una aspiración, como descripción de las características del oficio de enseñar, o como discusión sobre las peculiaridades o limitaciones con que tal condición se da en los docentes. El carácter polémico del profesionalismo obedece a que -como todos los temas en educación- está lejos de ser 
ingenuo, o desprovisto de argumentos interesados y de agendas más o menos ocultas. Expresiones como la de profesión -así como la de calidad y muchas otras que circulan en el campo educativo- "esconden en su seno opciones y visiones del mundo, arropadas en imágenes que normalmente se viven como positivas y deseables, y que es necesario desvelar si queremos ir en este análisis más allá de las primeras impresiones" (Contreras, 2011, p. 17).

Diferentes autores (Apple, 1990; Densmore, 1990; Ginsburg, 1990; Martínez Bonafé, 1999, entre otros) en relación con los niveles primarios y secundarios de educaciónsostienen que el trabajo docente ha ido sufriendo una sustracción progresiva de una serie de cualidades que ha conducido a los enseñantes a la pérdida de control y sentido sobre el propio trabajo. ${ }^{5}$ Afirman que la creencia de los profesores en su carácter de 'profesionales' tiene como eje una preocupación por el estatus y los privilegios en cuanto a otros empleados, obviando cuestiones como las condiciones en las que desempeñan su trabajo. Para Densmore (1990, p. 128) "la ideología del profesionalismo representa, principalmente, una respuesta defensiva de los profesores a lo que objetivamente son unos procesos y unas condiciones laborales alienantes". Por un lado, "el mantener la apariencia de un grado significativo de autonomía en el desempeño del trabajo, legitima las limitaciones que se imponen al trabajo del profesor" (Densmore, 1990, p. 131). Por otro, "legitima y refuerza los elementos de la proletarización, tales como el trabajo intensivo" (Densmore, 1990, p. 139). De este modo, la ideología del profesionalismo acaba convirtiéndose en una forma de control. ${ }^{6}$ Según Ginsburg (1990, p. 81), dicha ideología "distorsiona, a la vez que refleja parcialmente, la realidad social, y sirve para impulsar o para detener la acción individual o colectiva, de forma que sirva a los intereses de determinados grupos sociales".

Otros autores (Lawn y Ozga, 1988 en Contreras 2011), desde una posición de la proletarización más matizada, han planteado que la estrategia del profesionalismo ha defendido imágenes positivas y ha agrupado al profesorado en torno a reivindicaciones y luchas en su intento social por construir una cualificación. En este sentido, plantea Contreras (2011, p. 25):

\footnotetext{
5 Fundamentalmente, la introducción de principios de "gestión científica del trabajo" ha generado transformaciones en las condiciones de trabajo del colectivo docente que han llevado a una racionalización del mismo -caracterizada por la división entre concepción y ejecución, descualificación y pérdida de control- e intensificación -junto a una mayor burocratización de las tareas-.

${ }^{6}$ Susan Robertson $(1996,2012)$ señala que la promesa del profesionalismo de mayores grados de autonomía encubre nuevas formas de control a los docentes y advierte que se asiste a una forma de gobernanza global del trabajo de los docentes.
} 
No necesariamente hay que entender que el profesionalismo responde a un autoengaño o a una forma de control sobre el profesorado. Como tampoco hay por qué entenderlo como una estrategia para la mejora del status. Tal y como lo expresan estos autores, las demandas profesionales también podrían representar el compromiso con una ética del oficio.

Si bien la adopción de posiciones más moderadas sobre el profesionalismo, como es el caso de la planteada por Lawn y Ozga (1988), evitan caer en los reduccionismos propios de las posiciones extremas, aquí se reconoce la potencialidad de la perspectiva de la proletarización como lente crítico para abordar la cuestión del trabajo docente en la universidad. Ante la omnipresencia de la ideología del profesionalismo, que ha acompañado los procesos de reforma derivados de la implantación del Espacio Europeo de Educación Superior -y que se han ido expandiendo a las latitudes del sur- los aportes de los teóricos de la proletarización sirven como forma de 'vigilancia epistemológica' para no perder de vista en el análisis, las condiciones de tipo estructural en las que dicho trabajo se desarrolla. Y en los casos que se utilice el concepto de 'profesión' -fundamentalmente con el afán de ser fiel a las categorías empleadas por autores que aquí se retoman- se lo hará teniendo presente las salvedades realizadas.

En función de lo dicho, y parafraseando a Martínez Bonafé (1999), en esta comunicación se considera que lo que hace el profesorado universitario en el ejercicio de su función docente, es trabajo. Es precisamente esta categoría la que permite dar cuenta, por un lado, de la experiencia cotidiana de profesores -y sus condiciones simbólicas y materiales- en el ejercicio de su función docente; y, por otro, de las condiciones estructurales más amplias -y en relación con otros campos- que la atraviesan.

Un acercamiento al concepto de trabajo puede hacerse recuperando los aportes de Karl Marx, en la medida que permite pensarlo como una actividad de transformación de la realidad y de los sujetos que la realizan. Para Marx, la noción de trabajo constituye una categoría antropológica que va más allá de su dimensión puramente económica. No considera el trabajo como un mero medio para la producción de mercancías, sino como la expresión de las capacidades físicas y mentales y el lugar en donde el hombre se desarrolla y perfecciona (o más bien, donde se debería desarrollar y perfeccionar) ${ }^{7}$. En 'La ideología

\footnotetext{
${ }^{7}$ A partir de su concepción de la naturaleza humana como la de un ser que solo puede encontrar su perfección en el trabajo, el tema central de su filosofía fue la transformación del trabajo sin sentido, enajenado, del trabajo
} 
Alemana', Marx y Engels (1970) plantean que es precisamente el trabajo, la capacidad de generar los propios medios de vida y así producir la propia vida material, lo que diferencia al hombre de los animales. En el volumen 1 de 'El Capital', Marx (1973) afirma que el hombre a través del trabajo actúa sobre la naturaleza exterior y la transforma, al tiempo que transforma su propia naturaleza, desarrollando las potencias que dormitan en él y sometiendo el juego de sus fuerzas a su disciplina. En este sentido, el trabajo constituye un proceso de transformación del yo, que en términos de Foucault podríamos denominar de 'subjetivación'.

En el caso del trabajo docente, puede decirse que requiere para su desarrollo una energía vital a la que le han incorporado conocimientos y destrezas específicos. Dicha cualificación -ese añadido a la energía vital humana que se identifica con el 'valor de uso'- es lo que adquiere 'valor de cambio' en el mercado de trabajo. El valor de cambio se encuentra regulado por la totalidad de trabajos socialmente necesarios para producir esa fuerza de trabajo. Martínez Bonafé (1999) menciona tres categorías de trabajos no específicos de una determinada actividad laboral: 1) los destinados a producir la energía física humana, 2) los destinados a la cualificación de la energía vital, de modo que se pueda desarrollar un trabajo específico, y 3) los destinados a la persuasión, esto es, a la conducción de las voluntades formalmente libres hacia las necesidades del proceso productivo.

En relación con la cualificación de la energía vital, Bourdieu y Passeron (1995) afirman que en el caso de los sistemas educativos, se trata de una cualificación destinada a garantizar una formación homogénea que permita reproducir determinada arbitrariedad cultural. Esta cualificación -inicial y permanente- de los agentes encargados de realizar el trabajo docente (el 'trabajo pedagógico' en términos de los autores franceses), se complementa con instrumentos de control tendentes a garantizar la ortodoxia frente a las 'herejías individuales'. Obtenida la cualificación destinada a dotar de valor de uso -para el comprador- y valor de cambio -para el vendedor- a la fuerza de trabajo, el profesorado la vende a cambio de un salario, adquiriendo aquella la forma de 'mercancía'. Así, el trabajo docente supone "realizar una actividad, en este caso con una supuesta cualificación, de tipo asalariado (...) [sometida] a las condiciones y a las exigencias establecidas por el que contrata" (Rivas Flores, 2000, p. 8). En el caso que se analiza, el del profesorado de las universidades públicas, el que contrata es el Estado y, por tanto, es en la lucha con este

como un mero medio, en un trabajo enriquecedor, libre. En sus primeros escritos, llamó "actividad personal” a la realización de la inclinación al movimiento propia del hombre. Más tarde cuestionó la forma concreta que adopta dicha actividad en las sociedades de explotación y, a partir de la diferenciación entre trabajo libre y trabajo enajenado, criticó la alienación y abogó por la emancipación del trabajo. 
actor donde se dirimen las condiciones salariales y donde se configuran las condiciones materiales y simbólicas, así como los modos de regulación del trabajo.

En cuanto al trabajo de persuasión, resultan relevantes los aportes de Martínez Bonafé (1999) en relación con "los motivos para que el docente quiera vender su fuerza de trabajo" y lo que Becher (2001) identificó, en el caso del campo universitario, como 'la fuerza motivadora para seguir adelante'. Para el primero de los autores, resulta necesario un trabajo específico tendiente a persuadir a los docentes para que se sometan a las necesidades y exigencias del sistema y para que -a pesar de situaciones de explotación- estén dispuestos a invertir sus esfuerzos y buscar satisfacción en su trabajo. Para el segundo de los autores, es 'la búsqueda de reconocimiento' lo que motiva el esfuerzo por realizar el trabajo académico. En palabras de Becher (2001, p. 77): "lo más importante que busca el académico no es el poder, tras el cual va el político, ni la riqueza, tras la cual va el hombre de negocios, sino la buena reputación". Se trataría, en términos de Bourdieu, de la puesta en juego de un capital de tipo simbólico, lo que genera el interés por invertir en el campo. ${ }^{8}$ El especialista francés en temas del trabajo, Christophe Dejours, quien al igual que Marx, entiende que el trabajo 'no es solo producir sino transformarse', destaca el papel de la 'retribución moral', esto es, el reconocimiento de los otros en dicha transformación.

En el 'Tomo I: Sexualidad y trabajo' de su obra 'Trabajo vivo', cuyo punto de partida es la pregunta por lo que le debe la subjetividad a la experiencia del trabajo, Dejours (2012) plantea que la experiencia del trabajo permite a quien la realiza, adquirir nuevas habilidades y descubrir nuevos registros de sensibilidad. Por la experiencia del trabajar, se aprende a conocer los propios límites, las torpezas, se amplía el repertorio de las impresiones afectivas y se descubren nuevos virtuosismos. De este modo, por el reconocimiento carnal del mundo y de sí mismo a partir de la experiencia del trabajo, la subjetividad crece y se transforma. En palabras de Dejours (2012, p. 148): "Trabajar no solamente es producir, también es poner a prueba el cuerpo, con la posibilidad de salir de ella más sensible que antes, de incrementar por lo tanto la capacidad propia de sentir placer". $Y$ dicha experiencia subjetiva del trabajo confronta al sujeto no solamente con la resistencia del mundo a nuestra voluntad -lo que se denomina lo 'real del trabajo'- sino también con la resistencia de la propia personalidad a evolucionar frente a la experiencia del trabajo -lo real del inconsciente-. $Y$ es que, sostiene Dejours (2012, p. 151), "trabajar es también, quiérase o no, confrontarse consigo mismo, en

\footnotetext{
${ }^{8}$ Sostiene Densmore (1990) que en la construcción de la motivación, como en la interiorización de la disciplina, la ideología del profesionalismo juega un papel importante como montaje tendiente a ocultar la realidad de cada situación de trabajo, valiéndose para ello de mecanismos como el control directo y la autonomía responsable.
} 
la desagradable forma del desfallecimiento, de la pérdida de control, del error o la equivocación". Trabajar, además de enfrentarse con resistencias externas -de lo real material- e internas -de lo real inconsciente- es toparse con las relaciones sociales de poder. Porque, como afirma Dejours (2012, p. 152) "el trabajo no se despliega solamente en el mundo objetivo y en el mundo subjetivo, también lo hace en el mundo social". $Y$ es precisamente considerando lo que implica el trabajo en términos de dichas confrontaciones, cuando se puede reconocer lo que representa el trabajo en tanto puesta a prueba de la subjetividad. De allí que, ante quienes anuncian el fin del trabajo, Dejours defienda su centralidad, tanto a nivel de los sujetos, como para la sociedad. Para Dejours (2000), el trabajo es un mediador irreemplazable entre sujeto y sociedad, no solo a título de analizador o de operador de inteligibilidad de las conductas humanas, sino también, a título de 'lo que está en juego' (enjeu) material y simbólicamente en las confrontaciones concretas entre subjetividades singulares y las relaciones de dominación. “'Lo que está en juego' que no sería en absoluto contingente, sino fundamental, en un sentido literal del término, para la reproducción y la evolución de la sociedad" (Dejours, 2000, p. 329).

En relación con su concepción del trabajo, en su libro 'L'évaluation du travail à l'épreuve du réel: Critique des fondements de l'évaluation', Dejours (2003) afirma que trabajar supone un acto dirigido hacia una meta de producción -que incluye los pensamientos que le son inseparables- cuya característica principal es que a pesar de que esté bien diseñado, su organización sea rigurosa y sus instrucciones y procedimientos sean claros, resulta imposible lograr una calidad en estricta conformidad con lo prescrito. $Y$ esto, afirma Dejours (2003), porque

las situaciones de trabajo ordinario están cargadas de acontecimientos inesperados, averías, incidentes, anomalías de funcionamiento, incoherencias organizacionales, imprevistos, provenientes tanto de 10 'material' de las herramientas y las maquinarias, como de los otros trabajadores, de los colegas, directivos, subordinados, del equipo, la jerarquía, incluso de los clientes. Hay que reconocer no se trata de un trabajo de aplicación. De hecho, siempre aparece un desfase entre lo prescrito y la realidad concreta de la situación ${ }^{9}$

\footnotetext{
${ }^{9}$ Por tratarse de un libro consultado en versión Kindle, no se ofrece número de páginas sino 'locations', que es la unidad de medida utilizada en tal formato. La cita fue extraída de la 'locations' 215/1439.
} 
Todos esos incidentes que vienen a 'perturbar' las previsiones y las predicciones, sostiene Dejours (2009), es lo que se denomina 'real'; todo trabajo está afectado por las irrupciones de la resistencia de lo real. En este sentido, trabajar consiste en llenar la distancia entre lo prescripto y lo efectivo y como lo que se debe hacer para llenar tal distancia no se puede prever con antelación, Dejours (2003) plantea que "el camino a seguir entre lo prescrito y lo real debe ser descubierto o inventado por el sujeto que trabaja"10. En su libro 'Trabajo vivo' sostiene que lo irreductible en el trabajo es la gestión del desfase entre lo prescrito y lo efectivo; es decir de todo lo que surge en situación real sin haber sido previsto por la concepción, la planificación y la organización de la tarea (Dejours, 2012).

\section{El trabajo docente como cruce de la profesión de origen y la docencia}

El abordaje de la docencia como trabajo supone detener la mirada en el 'hacer', en la práctica docente. Como se dijo, el análisis de la docencia como práctica social desde la categoría de trabajo, permite dar cuenta de las condiciones materiales y simbólicas en las que esta se desarrolla. La dimensión disciplinar constituye uno de los aspectos más relevantes que organizan la práctica docente en la universidad y juega también un papel fundamental en la configuración del 'ser' docente. 'Ser' y 'hacer' docente que son distinguidos aquí solo con fines analíticos, ya que en el quehacer cotidiano de quienes 'son' profesores y 'ejercen' la docencia en la universidad, ambas dimensiones resultan indisociables.

Una peculiaridad del campo universitario, es que allí se nomina como docente o profesor, a sujetos que -provenientes de mundos académicos diversos- tienen como común denominador ejercer la función docente. Se trata de miembros de las diferentes tribus que habitan la universidad, con sus particulares creencias, ídolos, modos de comunicación y organización, y formas específicas de división del trabajo. Así, biólogos, matemáticos, abogados y médicos, pero también pedagogos, trabajadores sociales e ingenieros, entre otros, son agrupados en una categoría ocupacional que tanto en sus prescripciones, regulaciones y mecanismos de evaluación es tratada de manera homogénea. $Y$ esto, sostiene Scott (2008), por razones de tipo administrativo (ya que hay instituciones con objetivos especiales que se han creado con este propósito), social (porque todas estas formas de docencia tienen - ¿o tenían?- en común la socialización de los jóvenes) y

\footnotetext{
${ }^{10}$ Traducción propia, ‘locations’ en libro versión Kindle: 212/1439.
} 
normativo (porque la mayoría de las formas de 'docencia' se fundamentaban en ideas e ideales de mejora, emancipación e instrucción).

Como señala Lucarelli (2004), la peculiaridad de la institución en la que se desarrollan las prácticas docentes connota con singularidad el proceso de construcción de la propia identidad. De allí que, en un contexto como el universitario, donde el conocimiento disciplinar es altamente valorado, sea necesario preguntarse por lo que define la situación de los docentes en la institución: ¿es la práctica de enseñar? o ¿la práctica específica para la cual fue formado en su campo particular? En general, el acceso al trabajo académico en la universidad está ligado a la obtención de un cargo docente. Es el ejercicio de tareas de enseñanza lo que justifica la presencia de docentes o profesores en la universidad, más allá de que realicen otras funciones y actividades. Sin embargo, no es por el desempeño o los saberes vinculados a este tipo de tareas, por lo que se es reconocido en la universidad, sino más bien por el nivel de preparación académica (conocimientos disciplinares) y el ejercicio de la profesión de origen. Ese tipo de saber especializado, específico de cada tribu -y no proveniente del campo de la docencia- se convierte en capital simbólico, esto es, en capital reconocido por los otros y por uno mismo. Como sostiene Lucarelli (2004, p. 508):

El docente universitario se reconoce a sí mismo por su profesión de origen y se identifica con el título otorgado por la unidad académica donde se graduó. La legitimidad profesional de sus prácticas como médico, ingeniero, bioquímico, contador, es indiscutida, se origina en un saber acreditado escolarmente y cuenta con mayor o menor grado de aprobación, según cómo se hayan dado las negociaciones y las luchas curriculares, en el seno de la comunidad profesional respectiva. El poder y el prestigio no provienen de la docencia universitaria como saber pedagógico, sino del dominio de un campo científico, tecnológico o humanístico determinado.

Desde un punto de vista histórico, la tensión entre la disciplina y la docencia puede entenderse en el marco del proceso de expansión y diferenciación que, a partir de la segunda mitad del siglo XX, atravesaron los sistemas de educación superior a nivel mundial. Hasta ese momento, en la universidad de élite, el trabajo docente, y específicamente la práctica de enseñanza, constituía una sin requerimientos de formación específica. Así, "asociada a procesos de transmisión en sentido reproductivo, con el recaudo de cierta fidelidad epistemológica a las disciplinas y campos de conocimientos de pertenencia de las mismas, solo suponía el dominio de los contenidos pertinentes” (Edelstein, 2012, p. 10). La 
masificación de las universidades -expresadas en las cifras indicativas de número de estudiantes por cargos docentes, pero también en la diversidad social y cultural de las poblaciones ingresantes- exigió la expansión del cuerpo docente, el cual se convirtió en una ocupación atractiva para aquellos graduados con dificultades para insertarse en el mercado ocupacional, ya sea por características de sus profesiones, por el fracaso en opciones profesionales de corte liberal, o por la falta de dinamismo del propio mercado laboral. También resultó una opción sugerente para aquellos que vieron en la docencia la 'puerta de entrada' a la universidad -y la posibilidad de desarrollar las actividades de su interés, por ejemplo, la investigación. De esta manera, en muchos casos, la universidad se convierte en un refugio ocupacional y no en una elección 'vocacional', llegándose a ella por 'descarte'. En la medida en que la docencia universitaria, está formada por graduados de todas las disciplinas y muy especialmente de aquellas cuyo campo ocupacional extrauniversitario resulta más recortado y complejo, constituye una 'categoría residual' (Chiroleu, 2002). A esta situación también se refiere Edelstein (2012) al mencionar la categoría de 'profesión académica'. Sostiene que tal denominación alude a la situación de aquellos graduados que, una vez obtenidas sus titulaciones, no ejercen la profesión para la que fueron formados más allá del espacio universitario, sino que desarrollan toda su trayectoria profesional y laboral en las propias universidades. Y, aún en el supuesto de la probable dedicación a la investigación en ciertos casos, la mayoría concreta su carrera en una especialidad a partir de la obtención de cargos docentes, por lo que la enseñanza se torna, al margen de sus intereses prioritarios, en actividad nuclear. Ante esto, plantea la autora:

Sin considerar en términos de positividad o negatividad esta característica, de hecho la docencia en las universidades -en tanto garante de permanencia- se constituye en pilar de constitución identitaria en lo profesional para un número importante de universitarios. Se explicite o no, esto puede significarse también -al menos en términos conjeturales- como una clave de interpretación, en la tendencia a una creciente interpelación a las prácticas de enseñanza así como una mayor valorización de la docencia y la formación necesaria para la misma, también en las universidades" (Edelstein, 2012, p. 11).

Lo planteado da cuenta del papel que desempeñan las disciplinas en la configuración de la docencia en particular y el trabajo académico en general. La heterogeneidad, diversidad y diferenciación, productos de la presencia de campos disciplinares específicos, 
da lugar a posiciones que oscilan, según Becher (1993, 2001), entre dos polos: el que entiende la práctica académica -y la docencia como parte de ella- como un espacio construido más allá de las disciplinas de origen, y el que, por el contrario, deposita en los conocimientos específicos el papel determinante de la identificación ocupacional. En este sentido, resulta relevante pensar la docencia universitaria como un lugar de encuentro entre dos campos: el de la docencia y el de las profesiones de origen, los cuales son muy diversos (Souto, 1996).

Clark (1983) también señala la peculiaridad de lo que denomina 'profesión académica'. Sostiene que la situación de dicha profesión es fundamentalmente distinta de la de cualquier otra, en la medida que en otros conjuntos organizacionales amplios, prevalece un tipo ocupacional dominante (por ejemplo, los médicos en los centros de salud). Sin embargo, en la universidad se suele aplicar la etiqueta general de 'profesor' a diversos núcleos de académicos: a los abogados, los arquitectos, los ingenieros, los pedagogos, los biólogos, etc. La fragmentación del trabajo académico constituye una singularidad del campo universitario; antes que un grupo fuertemente cohesionado de profesores con una visión única del mundo, lo que se presenta es la coexistencia de múltiples grupos con adscripciones disciplinares diversas.

El mundo universitario no constituye un espacio homogéneo. En la construcción de la heterogeneidad que lo caracteriza, la dimensión disciplinaria ocupa un papel relevante. Cada disciplina configura formas propias de socialización a quienes pretenden formar parte de ella, que plantea sus propias lógicas de pensamiento, establece sus rituales, sus ídolos, etc. Ingresar a la universidad en carácter de estudiante o de docente supone socializarse no solo en una cultura institucional peculiar, sino también en una determinada cultura académica. Y aunque se haya transitado varios años como estudiante en una determinada carrera universitaria, que brinda las herramientas necesarias para la apropiación de los saberes de la cultura disciplinar de pertenencia, el pasaje a la categoría de 'docente' implica nuevos aprendizajes en relación con la lógica de la disciplina. Es decir, ser profesor de determinada área disciplinar no constituye una extensión de las prácticas ejercidas y de las identidades construidas durante la experiencia como estudiante. Los procesos de aprendizaje y afiliación -tanto cognitiva como institucional- (Coulon, 1997) que sirvieron para la incorporación del estudiantado a ese nuevo mundo que es la universidad, necesitan ser puestos en marcha nuevamente para habitar el mundo universitario desde otro lugar: el de profesor. Ser docente universitario implica nuevas y diferentes relaciones con la institución, con el estudiantado, 
con aquellos que de maestros pasan a ser colegas. Pero también, supone nuevas relaciones con un conocimiento que ahora debe ser enseñado. $Y$ las formas de enseñanza -dada la naturaleza de ese conocimiento, entre otras cosas- no son las mismas para un profesor de biología que para uno de comunicación, tampoco para uno de ingeniería en relación con uno de arte, así como tampoco son las mismas las herramientas con las que se cuenta para llevar a cabo dicha tarea. En muchas áreas, los profesores cuentan fundamentalmente con la solvencia en el conocimiento de su disciplina. Son más bien intuiciones las que se tienen acerca de las características de sus estudiantes y las peculiaridades de los procesos de aprendizaje, así como de las limitaciones y potencialidades de determinadas herramientas o estrategias de enseñanza. En otras áreas disciplinares, sin embargo, se considera indispensable tener estos conocimientos para llevar adelante la práctica de enseñanza. Estas son fundamentalmente aquellas áreas en las que el sujeto de aprendizaje o la práctica de enseñanza forman parte de su objeto de estudio. De modo que en relación con la formación pedagógica, los profesores universitarios se encuentran en desigualdad de condiciones, pues muchos no fueron formados para ser docentes, sino para desempeñarse como biólogos, ingenieros, médicos, contadores, etc. (Walker, 2013).

La atención a la dimensión disciplinaria de la universidad en relación con el trabajo docente, no significa solo diferencias en cuanto a los saberes y herramientas para llevar adelante la práctica de enseñanza. Como construcciones socio-históricas, las disciplinas también ubican diferencialmente a sus integrantes en el espacio universitario, suponen condiciones de desigualdad en términos de reconocimiento y prestigio. Socialmente, no es lo mismo ejercer el trabajo docente siendo médico que pedagogo. No goza del mismo prestigio un profesor del área de Ciencias Exactas y Naturales o Medicina, que otro de Ciencias Sociales, Humanidades o Arte. Los mecanismos de cierre social de determinadas comunidades profesionales y su auto-legitimación mediante la ideología técnicofuncionalista, que argumenta la necesidad de una larga y selectiva formación especializada, se erigen como 'estrategias de protección' del gremio (Collins, 1989), que generan representaciones sociales sobre tales comunidades.

Por último, las condiciones del trabajo docente son diferentes entre aquellos que se desempeñan en áreas con perfil profesional o académico. Muchas veces, este perfil se materializa en las dedicaciones docentes. En las carreras de corte profesional prevalecen las dedicaciones docentes simples y los profesores asociados que desarrollan su principal actividad profesional fuera de la universidad. Esto también supone diferencias en cuanto a 
las condiciones de trabajo docente. No es lo mismo que el trabajo en la universidad constituya la principal o exclusiva actividad de un profesor, a que sea una labor secundaria que ocupa una pequeña parte del tiempo de trabajo.

\section{La docencia como práctica compleja}

La revisión de la bibliografía especializada en el tema de la docencia, permite advertir la recurrente referencia al carácter complejo de dicha práctica. Los estudios especializados en docencia universitaria también apelan a tal calificativo. Sin embargo, dicha expresión puede resultar una frase trillada. En ocasiones sirve de comodín para aquello que resulta difícil explicar. $Y$ por lo general, no se lo define, ya que se presume la univocidad del concepto. Morín (1994) plantea que existe complejidad toda vez que se produzca un enmarañamiento de acciones, de interacciones, de retroacciones, y en la que exista algún elemento aleatorio. Es en referencia a ambas cuestiones, que aquí se considera la docencia como una tarea compleja.

En tanto 'enmarañamiento de acciones, interacciones y retracciones' la 'docencia' es y ha sido siempre, como señala Scott (2008), una etiqueta que cubre una gama heterogénea de actividades eclécticas, aunque en los debates de tipo normativo, a menudo ha acabado por sucumbir y se ha convertido en una categoría homogénea y no problemática. Sostienen Martínez, Gretter, Lagatta y Vai (2009, p. 137), que la complejidad de la docencia universitaria "solo se advierte cuando lo vivido a diario se problematiza o se interpela" permitiendo reconocer su multi-dimensionalidad. Una posible manera de abordar la complejidad y peculiaridad de la docencia universitaria, es considerando las dimensiones prescripta y real del trabajo (Dejours, 1998). Por 'prescripto' se entienden las 'tareas' que se deben desarrollar, aquello que se desea obtener, o lo que se debería hacer. Lo 'real' hace referencia a las 'actividades' que se hacen para tratar de alcanzar los objetivos fijados por la tarea. En este sentido, el trabajo docente constituye la actividad desplegada por hombres y mujeres para enfrentar lo que en una tarea utilitaria no puede obtenerse mediante la estricta ejecución de la organización prescripta (Dejours, 1998). Esto permite considerar la actividad real del docente universitario, lo desplegado en el día a día, en el hacer cotidiano (Martínez et al, 2009, p. 152); aquello que "se nos escapa y se convierte a la vez en un enigma a descifrar" (Tello et al, 2009, p. 255). Las actividades que los profesores desarrollan en el ejercicio de su función docente, exceden las tareas directamente relacionadas con la enseñanza. Como sostienen Tello et al (2009, p. 249) "la práctica docente como trabajo está 
identificada social e individualmente por la práctica pedagógica, pero su alcance es mucho mayor ya que involucra una compleja red de actividades y relaciones sociales y políticas que la traspasan". Entre ellas, actividades de asesoramiento, tutorías, dirección de "tesistas", formación de futuros o nuevos docentes, tareas de gestión relacionadas con la docencia, investigaciones vinculadas con la enseñanza, diseño y producción de materiales didácticos, por mencionar algunas. Pero, si se consideran las demás funciones de la universidad, es posible reconocer otras dimensiones en las que se encuentra comprometida la actividad del profesorado a escala institucional (Miguel Díaz, 1998). Como sostiene Souto (2010, p. 326):

Los que enseñan a los estudiantes son también sujetos de la gestión, de la producción de conocimiento y de la transferencia en la comunidad, se ocupan de identificar y formar a los que van a garantizar continuidad y renovación de la vida institucional. Son los mismos que pelean por cuotas de poder para sostenerse y sostener su tarea, para proteger o cambiar un proyecto institucional, los que enfrentan cotidianamente la tentación de la conducta desviada... Los que, como si todo eso fuera poco, sufren en sí el entrecruzamiento imprevisible de todo esto con su extracción de clase, con su género, con su etnia, con las propias y singulares líneas de su biografía.

Más allá de ensayar una posible lista de actividades implicadas en el ejercicio de la función docente del profesorado universitario, el intento de determinarlas resulta una difícil tarea, ya que varían según el momento histórico, las características de la institución, su historia, el campo disciplinar al que se pertenece, etc. Como sostiene Lucarelli (2004, p.505), "el reconocimiento de la complejidad del contexto, en el que se entrecruzan estructuras científicas, sociales y educativas en proceso de cambio, es el que permite entender a la profesión docente universitaria como un algo complejo y no uniforme".

En relación con la segunda de las cuestiones involucradas en el concepto de complejidad, la existencia de 'elementos aleatorios', puede decirse que si hay algo que describe las prácticas docentes es su naturaleza incierta. En el caso particular de la enseñanza, una peculiaridad que atraviesa dicha práctica es la falta de certeza y el carácter no predecible de la relación entre enseñanza y aprendizaje.

\section{Reflexiones finales}

En función de lo planteado, el trabajo docente universitario, como actividad cotidiana (Heller, 1987), constituye una práctica social heterogénea, múltiple e incierta, que es vivida y 
percibida diferencialmente por los docentes, en función de sus particulares posiciones en el espacio universitario. Supone la coexistencia de experiencias de trabajo desiguales (Sennet, 2006), investidas de una aparente uniformidad de exigencias y condiciones (Dejours, 2009). Si bien la diversidad y singularidad de las experiencias de este tipo particular de trabajo sobre los otros, se resisten a ser formalizadas (Dubet, 2006), pueden señalarse situaciones que las atraviesan y, aunque adopten formas particulares según cada caso, se hacen presentes en el trabajo docente cotidiano del profesorado. Muchas de estas situaciones devienen de los cambios acaecidos en el mundo del trabajo en general, que se hacen presentes en el campo universitario, atravesando las experiencias singulares de los docentes y otorgándole complejidad a sus prácticas cotidianas. Por otra parte, el trabajo docente universitario no escapa al actual clima de época, en el que todo es evaluable y lo que no, es sospechoso de mediocridad u oscurantismo. Desde las últimas décadas del siglo XX, los docentes han sido objeto de crecientes evaluaciones de desempeño tendientes a posicionarlos en el espacio universitario, otorgarles retribuciones económicas u orientar su tarea hacia propósitos de mejora. A través de formas de evaluación homogéneas y homogeneizantes y confundiendo intencionalmente el trabajo con sus resultados, los mecanismos hegemónicos de evaluación del campo universitario no son capaces de dar cuenta de las especificidades del trabajo docente, porque el carácter no observable de los procesos en él implicados, se resiste a ser 'medido' con 'evaluaciones objetivas', y esto porque, como sostiene Dejours (2009), lo esencial del trabajo es esencialmente invisible e inconmensurable.

\section{Referencias}

Apple, Michael. (1990). Trabajo, enseñanza y discriminación sexual. En Thomas Popkewitz (Ed.) Formación del profesorado: tradición, teoría y práctica (pp. 55-78). Valencia: Universitat de València, Servei de Publicacions.

Becher, Tony. (1993). Las disciplinas y la identidad de los académicos. Pensamiento Universitario, 1(1), 56-77.

Becher, Tony. (2001). Tribus y territorios académicos. La indagación intelectual y las culturas de las disciplinas. Barcelona: Gedisa.

Bourdieu, Pierre. (2008). Homo Academicus. Buenos Aires: Siglo XXI Editores.

Bourdieu, Pierre y Passeron Jean-Claude. (1995). La reproducción. Elementos para una teoría del sistema de enseñanza. México: Fontamara. 
Chiroleu, Adriana. (2002). La profesión académica en Argentina. Boletín PROEALC, síntesis especial América Latina, mayo. Recuperado de http://www.proealc.uerj.br/documentos/revista synthesis/la profesion academica en argentina.pdf

Clark, Burton. (1983). El sistema de educación superior: una visión comparativa de la organización académica. México: Nueva Imagen/Universidad Futura/UAM.

Collins, Randall. (1989). La sociedad credencialista. Sociología histórica de la educación y la estratificación. Madrid: Ediciones Akal.

Contreras, José. (2011). La autonomía del profesorado. Madrid: Ediciones Morata.

Coulon, Alain. (1997). Le métier d'étudiant: l' entrée dans la vie universitaire. Paris: Presses Universitaires de France.

Dejours, Christophe. (1998). El factor humano. Buenos Aires: Lumen/Humanitas.

Dejours, Christophe. (2000). Travail, souffrance et subjectivité. Sociologie du travail, 42(2), 329-340.

Dejours, Christophe. (2003). L'évaluation du travail à l'épreuve du reel: Critique des fondements de l'évaluation. París: INRA Editions.

Dejours, Christophe. (2009). El desgaste mental en el trabajo. Madrid: Editorial Modus Laborandi.

Dejours, Christophe. (2012). Del trabajo a la subjetividad. En Trabajo Vivo (Tomo I, Sexualidad y trabajo). Buenos Aires: Editorial Topía.

Densmore, Kathleen. (1990). Profesionalismo, proletarización y trabajo docente. En Thomas Popkewitz (Ed.), Formación del profesorado: tradición, teoría y práctica (pp. 119-147). Valencia: Universitat de València, Servei de Publicacions.

Dubet, Francois. (2006). El declive de la institución. Profesiones, sujetos e individuos en la modernidad. Barcelona: Editorial Gedisa.

Edelstein, Gloria. (2012). Un debate vigente y necesario: la formación docente en las universidades. En María del Carmen Lorenzatti (coord.), Construcción cooperativa de políticas y estrategias de formación de docentes universitarios en la región (pp. 9-16). Córdoba: Universidad Nacional de Córdoba.

Ginsburg, Mark. (1990). Reproducción, contradicción y conceptos del profesionalismo: el caso de los futuros profesionales. En Thomas Popkewitz (Ed.), Formación del profesorado: tradición, teoría y práctica (pp. 80-118). Valencia: Universitat de València, Servei de Publicacions.

Heller, Agnes. (1987). Sociología de la vida cotidiana. Barcelona: Ediciones Península.

Lawn, Martin y Ozga, Jenny. (1988). ¿Trabajador de la enseñanza? Nueva valoración de los profesores. Revista de Educación, (285), 191-215. 
Lucarelli, Elisa. (2004). Prácticas innovadoras en la formación del docente universitario. Revista Educaçao, 27(3), 503-524.

Martínez, Deolidia, Amieva, Rita, Gretter, Silvia, Lagatta, Silvana, Vai, Dora. (2009). Subjetividad y trabajo docente en la Universidad. Fundamentos en Humanidades, 20(2), 135-160.

Martínez Bonafé, Jaume. (1999). Trabajar en la escuela. Profesorado y reformas en el umbral del siglo XXI. Madrid: Miño y Dávila Editores.

Marx, Karl. (1973). El Capital (Tomo 1: El proceso de producción capitalista). México: Fondo de Cultura Económica.

Marx, Karl y Engels, Friedrich. (1970). La ideología alemana. Barcelona: Grijalbo.

Miguel Díaz, Mario de. (1998). La evaluación del profesorado universitario. Criterios y propuestas para mejorar la función docente. Revista de Educación, (315), 67-83.

Morin, Edgar. (1994). Epistemología de la complejidad. En Dora Fried Schnitman (comp.), Nuevos paradigmas, cultura y subjetividad (pp. 421-442). Buenos Aires: Editorial Paidós.

Rivas Flores, Ignacio. (2000). El trabajo de los docentes. Jornadas Profesorado y Reforma (pp. 4-6). Libro de Comunicaciones. Grupo de Investigación HUM 619. Málaga, España.

Robertson, Susan. (1996). Teachers' work, restructuring and postfordism: constructing the new 'professionalism'. In Andy Hargreaves and Ivor Goodson (Eds.), Teachers' professional lives (pp. 28-54). London/Washington DC: Falmer Press.

Robertson, Susan. (2012). Teachers' Work, Denationalisation, and Transformations in the Field of Symbolic Control: A Comparative Account. Bristol: Centre for Globalisation, Education and Societies, University of Bristol. Recuperado de http://susanleerobertson.files.wordpress.com/2012/07/2012-robertson-teachersdenationalisation.pdf

Scott, Peter. (2008). ¿Divergencia o convergencia? Las relaciones entre docencia e investigación en la educación superior de masas. En Ronald Barnett (Ed.) Para una transformación de la universidad. Nuevas relaciones entre investigación, saber y docencia (Capítulo 4). Barcelona: Editorial Octaedro.

Sennett, Richard. (2006). La cultura del nuevo capitalismo. Barcelona: Editorial Anagrama.

Souto, Marta. (1996). Formación de profesores universitarios: condiciones para la formulación de una carrera docente. Iglú, (11), 17-26.

Souto, Marta. (2010). Panel 5. I Coloquio Iberoamericano La evaluación de la docencia universitaria y no universitaria: Retos y perspectivas. Revista Iberoamericana de Evaluación Educativa, 3(1e), 339-343. 
Tello, Ana María, Dequino, María Cristina, Delbueno, Horacio, Silvage, Benegas, Isidoro, Carlos, Romero, Marcelo, Jofré, José y Berraondo Marcos, María Rosa. (2009). Trabajo docente en la universidad. Regulaciones, subjetividad y sentidos, inscriptos en los ciclos de una investigación. Fundamentos en Humanidades, 10(II-20), 241-264.

Walker, Verónica. (2013). Evaluación y trabajo docente en la universidad: políticas, prácticas y actores. Estudio de casos en Argentina y España. (Tesis Doctoral). Universidad de Málaga, Málaga, España.

Recuperada

de

http://riuma.uma.es/xmlui/handle/10630/6868) 